\title{
Physical inactivity post-stroke: a 3-year longitudinal study
}

\section{Dorit Kunkel, Carolyn Fitton, Malcolm Burnett \& Ann Ashburn}

To cite this article: Dorit Kunkel, Carolyn Fitton, Malcolm Burnett \& Ann Ashburn (2015)

Physical inactivity post-stroke: a 3-year longitudinal study, Disability and Rehabilitation, 37:4, 304-310, DOI: $10.3109 / 09638288.2014 .918190$

To link to this article: $\underline{\text { http://dx.doi.org/10.3109/09638288.2014.918190 }}$

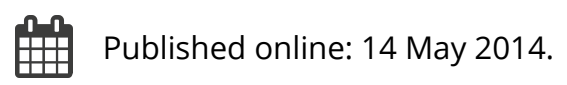

Submit your article to this journal $\widetilde{ }$

Llll Article views: 250

Q View related articles ¿

View Crossmark data ¿ 


\title{
Physical inactivity post-stroke: a 3-year longitudinal study
}

\author{
Dorit Kunkel, Carolyn Fitton, Malcolm Burnett, and Ann Ashburn \\ Faculty of Health Sciences, University of Southampton, Southampton General Hospital, Southampton, UK
}

\section{Abstract}

Purpose: To explore change in activity levels post-stroke. Methods: We measured activity levels using the activPAL ${ }^{\mathrm{TM}}$ in hospital and at 1,2 and 3 years' post-stroke onset. Results: Of the 74 participants (mean age 76 (SD 11), 39 men), 61 were assessed in hospital: 94\% of time was spent in sitting/lying, $4 \%$ standing and $2 \%$ walking. Activity levels improved over time (complete cases $n=15)$; time spent sitting/lying decreased $(p=0.001)$; time spent standing, walking and number of steps increased ( $p=0.001, p=0.028$ and $p=0.03$, respectively). At year $3,18 \%$ of time was spent in standing and $9 \%$ walking. Time spent upright correlated significantly with Barthel ( $r=0.69$ on admission, $r=0.68$ on discharge, both $p<0.01)$ and functional ambulation category scores ( $r=0.55$ on admission, 0.63 on discharge, both $p<0.05)$; correlations remained significant at all assessment points. Depression (in hospital), left hemisphere infarction (Years 1-2), visual neglect (Year 2), poor mobility and balance (Years 1-3) correlated with poorer activity levels. Conclusion: People with stroke were inactive for the majority of time. Time spent upright improved significantly by 1 year post-stroke; improvements slowed down thereafter. Poor activity levels correlated with physical and psychological measures. Larger studies are indicated to identify predictors of activity levels.

\section{- Implications for Rehabilitation}

- Activity levels (measured using activPAL ${ }^{\mathrm{TM}}$ activity monitor), increased significantly by 1 year post-stroke but improvements slowed down at 2 and 3 years.

- People with stroke were inactive for the majority of their day in hospital and in the community.

- Poor activity levels correlated with physical and psychological measures.

- Larger studies are indicated to identify the most important predictors of activity levels.
\end{abstract}

\section{Keywords}

Activity levels, standing, stroke, walking

\section{History}

Received 24 July 2013

Revised 11 April 2014

Accepted 22 April 2014

Published online 14 May 2014

\section{Introduction}

Regaining functional independence and resumption of walking and previously valued activities is a key aim of individuals participating in stroke rehabilitation [1]. Physical inactivity poststroke has been associated with decreased ability to perform activities of daily living, decreased muscle strength and cardiovascular fitness and a heightened risk for recurrent stroke and cardiovascular disease [2,3]. Although the importance of physical activity on health, well-being and stroke prevention are well established [3-6], people with stroke are generally de-conditioned, sedentary and less active than age matched healthy controls [7-14].

Objective monitoring devices such as pedometers and accelerometers are increasingly being used to obtain accurate measurements of physical activity levels. Various types of

Address for correspondence: Dr. Dorit Kunkel, PhD, Senior Research Fellow, Faculty of Health Sciences, University of Southampton, Mail point 886, Level E Centre Block, Southampton General Hospital, Tremona Road, Southampton SO16 6YD, UK. Tel: (0238120) 8669. Fax: (0238120) 4340. E-mail: dh197@ soton.ac.uk accelerometer are on the market [15-17] and have been used to measure components of physical activity, such as step count, minutes of activity, intensity of activity and frequency of postural transitions [18]. Pedometers are the least expensive option but they have shown to become less accurate at slower walking speeds [19] and hence are less suitable for assessing steps and walking in people with stroke.

Generally, the more technically complex the accelerometer that is used, the greater the number of postures that can be distinguished but the more complex systems are often expensive, sometimes "oversensitive", (recording erroneous steps during non-ambulatory movements or vibrations) and also less user friendly due to the requirements of fitting two or more sensors and the associated cabling [15,20,21].

The activPAL ${ }^{\mathrm{TM}}$ physical activity logger (PAL Technologies Ltd., Glasgow, UK) is one example of a newer class of monitoring devices that does not require cabling. This device contains a uniaxial accelerometer and it is much smaller $(3.5 \times 5.3 \times 0.7 \mathrm{~cm}$; $20 \mathrm{~g}$ ) than some of the other available devices, e.g. the ActiGraph [22]. Also unlike other accelerometers (such as the ActiGraph and the Actica [23]), the activPAL is placed on the mid-line of the thigh, about a third of the way down between the hip to the knee by direct adhesion to the skin. A further advantage is that the 
activPAL provides a direct output of steps and the accelerometry counts can be easily accessed and downloaded in an Excel file.

The validity of the posture classification elements and the analysis algorithm is described in detail elsewhere [20,24-27]. Evidence from these studies suggests that it is valid and reliable in measuring step count and cadence over a range of walking speeds for healthy young and older adults. Data from older people (mean 79 years) using the activPAL (collected over a period of $7 \mathrm{~d}$ ), suggested that this group of people spent approximately $251 \mathrm{~min} / \mathrm{d}$ active (upright) of which $81 \mathrm{~min}$ was spent walking and took an average 6343 steps/d [28].

A systematic review of studies describing physical activity among people with stroke compared a variety of measures and the authors concluded that valid and reliable data can be obtained using accelerometry-based measures [29]. The activePAL activity monitor has been used successfully [30,31] but the majority of studies that reported on the actual amount of mobility activity undertaken by stroke patients at different stages during their recovery in hospital [6-8] and in the community [9-14] used different devices. Two of the hospital based studies [7,8] used observational methodologies and others used a variety of instrumented recording devices [6]. Time spent being active/ upright in the hospital setting varied between studies. In the earliest study [8] participants were standing for only $2.3 \%$ of their observations during the waking day, others reported that participants spent on average $8.3 \%$ of the therapeutic day upright [6] and in the third study participants spent $12.8 \%$ of the recording time either transferring or walking [7]. Average number of daily steps in a study using the activPAL [31], ranged from 3680 to 12039 at the baseline assessment. These data were based on individual stroke patients' results whose onset dates varied considerably (range 3-20 months). The conclusions that can be drawn from these small scale studies are limited, however all authors agreed that the reported activity levels were low in all these studies despite patients undergoing rehabilitation in specialised stroke units. Activity levels though remain low among community dwelling people with stroke [9-13]. Michael et al. [9-11] reported that people with stroke took on average between 2608 and 2837 steps/d. Others showed that people with stroke (7379 steps/d, $n=10$ ) walked less than controls (14730 steps/d, $n=10$ ) [14]. A more recent study [13] confirmed these findings. They reported that people with stroke performed 5308 fewer activity counts and spent $79 \mathrm{~min}$ less on their feet than healthy controls. Most of the studies to date have small sample sizes $(n=10-42)$ and explore activity levels at a single time point and have not been able to relate activity data to stroke type, severity, physical or psychological measures. This study was part of a larger longitudinal project exploring physical recovery post-stroke. The main research question for this part of the project was: Which factors influence activity levels in the first 3 years post-stroke? Our aim was to extend previous work by using the activPAL Activity monitor (a) to examine free-living physical activity of a larger sample of people with stroke, longitudinally, at four time points over a period of 3 years and (b) to explore activity levels in relation to other measures and factors that may impact on activity levels with the intention to undertake a predictive analysis.

\section{Methods}

\section{Study design and subjects}

Ethical approval was obtained from the Dorset Research Ethics Committee (REC no. 04/Q2201/91). The recruitment process for the main study has been described in detail elsewhere [32]. Consecutively admitted patients with stroke (admitted to the Royal Bournemouth and Christchurch NHS Foundation Trust between April 2005 and December 2006), were recruited as soon as possible after admission and written informed consent was obtained as soon as a potential participant was well enough. Four hundred and sixteen patients were recruited to the main study (324 were able to give informed consent, for 92 participants assent was obtained from relatives). All patients who were consented to the main study between January 2006 until December $2006(n=218)$ were also considered for inclusion in the activity monitoring study; using random allocation. At the point of giving consent to the main study, the assessor called the statistician to find out allocation for this participant. The computerised randomisation schedule was based on the number of recruits and assessments that had to be completed by the assessors, the available manpower as well as the available activity monitors. Using this method approximately every third person recruited to the main study during the specified time period was also consented for participation in the activity monitoring sub study $(n=86)$.

\section{Activity monitoring}

The activPAL ${ }^{\mathrm{TM}}$ system (PAL Technologies Ltd., Glasgow, UK) produces a real-time signal conveying thigh inclination and limb movement. The associated proprietary software classifies postures into three types: (1) lying/sitting, (2) standing or (3) walking. In addition, steps are recorded during upright activities only. In this study, the activity monitor was attached in the morning until late afternoon/early evening on weekdays only (for pragmatic reasons to coincide within the working hours of the researchers and availability of the activity monitors). The activity monitor was attached to the unaffected leg, which has been confirmed as the most reliable method of attachment in people with impaired function [30].

The first assessment took place in hospital as soon as possible post consent; the other assessments took place at 1,2 and 3 years post-stroke onset. Data were collected (using four activPAL units) by two assessors in hospital and until the end of the first-year follow-up period. At this point, a routine data check revealed problems with the data collected by one of the assessors. These problems were reported to the University of Southampton and a 4-year data investigation into allegations of research misconduct commenced. The assessor in question left the University in 2007 and the investigation was completed in January 2013. The University of Southampton up held in full the allegations and confirmed that serious misconduct occurred with a breach of academic integrity. This delayed publication of the study findings and seriously affected the amount data we were able to include for analysis in this article. The data modification involved the duplication of some of the computerised activity monitor data sets. These duplications were identifiable because they resulted in incorrect dates and times and identical activity profiles, something which would be impossible under normal conditions. As part of the investigation, an independent external computer expert checked the data sets and confirmed that all the modified data sets were correctly identified. All data sets have been checked and all replica data sets have been removed. We are confident that the data we have presented and analysed in this article is valid and of high quality. Data were excluded for four participants in hospital and 24 data sets had to be excluded at Year 1. No further data sets had to be excluded after this point but not all participants were able to complete the assessments. The reasons for missing data have been outlined in Figure 1.

\section{Other measures}

Information on factors that may have an impact on activity levels was collected. These included: stroke classification (based on the 


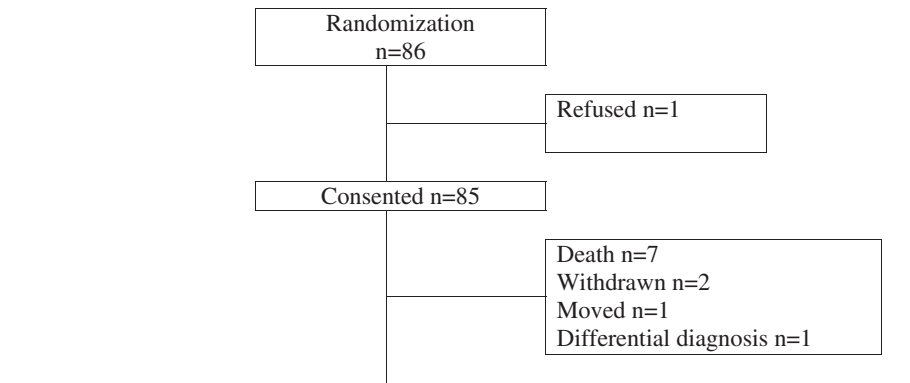

At least one assessment over time period $n=74$

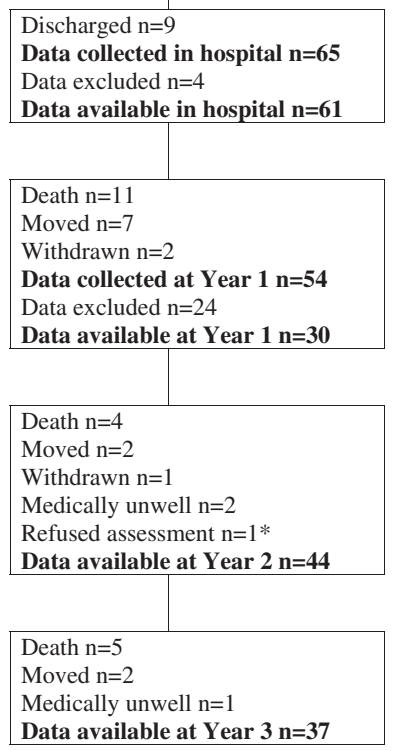

Figure 1. Flow chart outlining recruitment and drop out at each time point. *One participant refused the year 2 assessment but took part again at Year 3.

Oxfordshire Community Stroke Classification [OCSP]), length of hospital stay, Barthel score, Functional Ambulation Category (FAC), side of infarct, age, gender, functional mobility (Rivermead Mobility Index [RMI]), balance ability (Berg Balance Scale [BBS]), visual neglect (Star Cancellation Test $[\mathrm{SCT}]$ ) and mood (Hospital and Anxiety Depression Scale depression component [HADD]), see [32].

\section{Data analysis}

Descriptive analysis and inferential testing were completed using SPSS version 18 (SPSS, Chicago, IL). Descriptive statistics are presented for all cases. As the duration of activity monitoring differed significantly between time points $(p<0.01)$, activity monitoring data was converted into the percentage of time spent doing each activity for time spent in sitting/lying, standing and walking and into steps per min by recorded duration for number of steps, to allow statistical comparison and comparison to other studies. The intention was to undertake predictive analysis to identify the factors that have the greatest impact on activity levels. However, due to the large amounts of missing data at the different time points (see Figure 1 for reasons) and high degree of variability between participants, the number of complete cases was very small. Due to the small sample size and variability, we refrained from transforming the data in an attempt to normalise it and hence did not incorporate further in-depth or predictive analysis. Instead, we present raw data for complete cases (Table 3) and present correlations and differences in activity levels over time using Spearman correlations and related samples Friedman's two-way analysis of variance (ANOVA).

\section{Results}

Of the 86 participants who were randomly allocated to the activity monitoring sub-group, only 74 had at least one activity monitoring session during the study period. The reasons for missing data are presented in Figure 1. Results are presented for all cases $(n=74)$ and complete cases $(n=15$, participants who completed all of the activity monitoring assessments).

\section{All cases}

In total, 39 male and 35 female participants had an average age of 76 (ranging from 44 to 95 years) and completed at least one activity monitoring assessment. Twenty-two presented with a right hemisphere infarction, 44 with a left hemisphere infarction, for two participants infarction spread across both hemispheres and for six participants' this was not identified. Twenty-three participants were classified as PACI, 20 as LACI, 14 as PICH, 12 as POCI and 4 as TACI according to OCSP. Time post-stroke, length of activity monitoring sessions and descriptive data for activity levels are presented in Table 1. On average, the observation period ranged from 6 to $7 \mathrm{~h} / \mathrm{d}$. Although 85 people enrolled in the study, data were only available for 61 people in hospital, 30 people at year 1, 44 people at year 2 and 37 people at year 3, see Figure 1.

Sixty-one participants completed an activity monitoring session in hospital and this assessment took place on average $23 \mathrm{~d}$ after stroke onset. In hospital, participants spent approximately $94 \%$ of the recorded time in sitting and lying and only $4 \%$ and $2 \%$ in standing and walking ( $1 \mathrm{step} / \mathrm{min})$, respectively. At 1 year, less time was spent in sitting/lying (77\%) in favour of more time spent active in standing (16\%) and walking (7\%, 4 steps/min). Very little change in activity levels were observed at 2 years post-stroke (74\% of the time was spent sitting/lying, 17\% standing and $8 \%$ walking, 5 steps $/ \mathrm{min}$ ) and 3 years post-stroke (73\% sitting/lying, $18 \%$ standing, $9 \%$ walking, 5 steps $/ \mathrm{min}$ ).

\section{Complete cases}

For complete cases, a Friedman's two-way ANOVA analysis revealed significant decreases over time for percentage of time spent sitting/lying $(p=0.001)$ and increases in the time spent standing $(p=0.001)$. There was a small but significant change in the time spent walking $(p=0.028)$ and number of steps $(p=0.03)$. The number of steps (per minute of the recorded duration) increased steadily over the 3 years (from 2 steps/min in hospital, to 4 steps/min at year 1 and 5 steps/min at years 2 and 3 ), however, there was a high degree of variability between participants. The group median scores suggest continuing improvements in activity levels up to year 2 but this trend appeared to slow down or possibly plateaued at year 3 . We also examined the correlation between other measures and factors that may impact on activity levels, see Tables 2 and 3. In Table 2, we present the raw data for the 15 participants with complete activity data. In Table 3, we highlight activity levels in relation to participants' results on other measures.

Correlational analysis revealed that in this study activity levels did not correlate with age, gender, OCSP stroke classification or length of hospital stay (data not shown) at any time point. In contrast, activity levels correlated with Barthel scores and FAC scores at all time points. The time spent upright/active in hospital correlated significantly with Barthel $(r=0.69$ both on admission and $r=0.68$ on discharge both $p<0.01)$ and FAC scores $(r=0.55$ on admission and 0.63 on discharge, both $p<0.05$ ). These correlations remained significant at Year 1-3 with similar $r$-levels (not shown). These data (Table 3) highlight that those who achieved better Barthel and FAC scores were more active in comparison to those with poorer Barthel and FAC scores. 
Table 1. Descriptive data of activity levels for all available and complete cases at each assessment point.

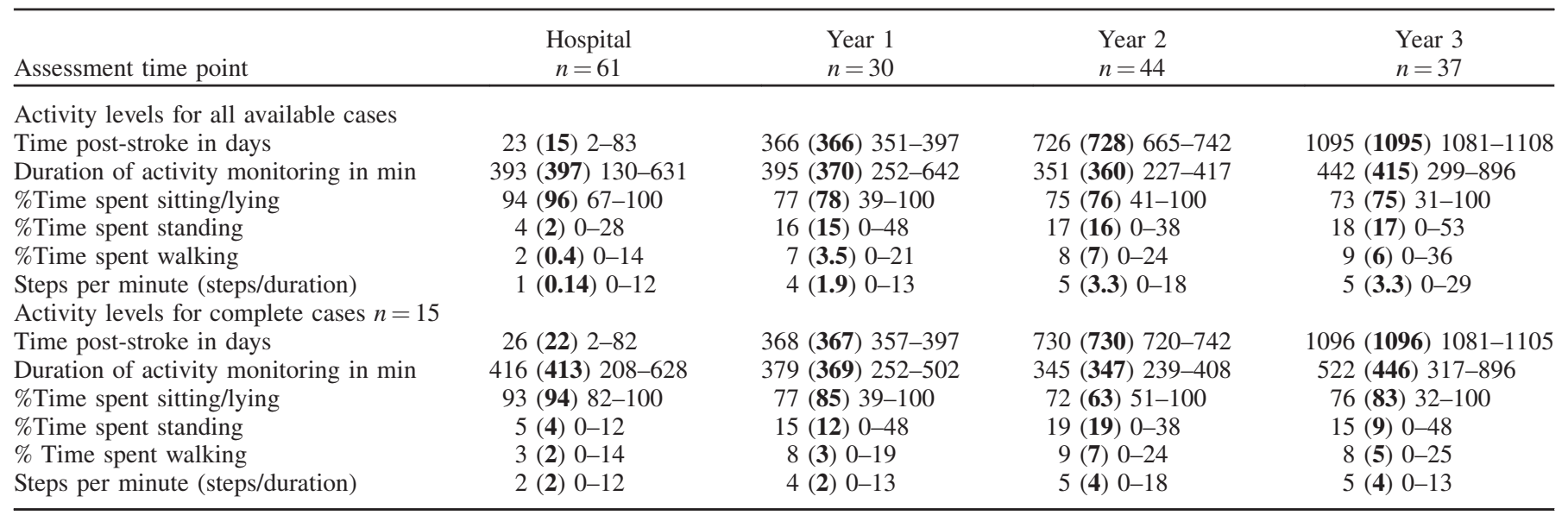

Results are displayed as Mean (median) min-max. Median values are in bold.

Table 2. Descriptive data (actual scores) for cases who completed all of the activity monitoring sessions (Hospital, Year 1, Year 2 and Year 3).

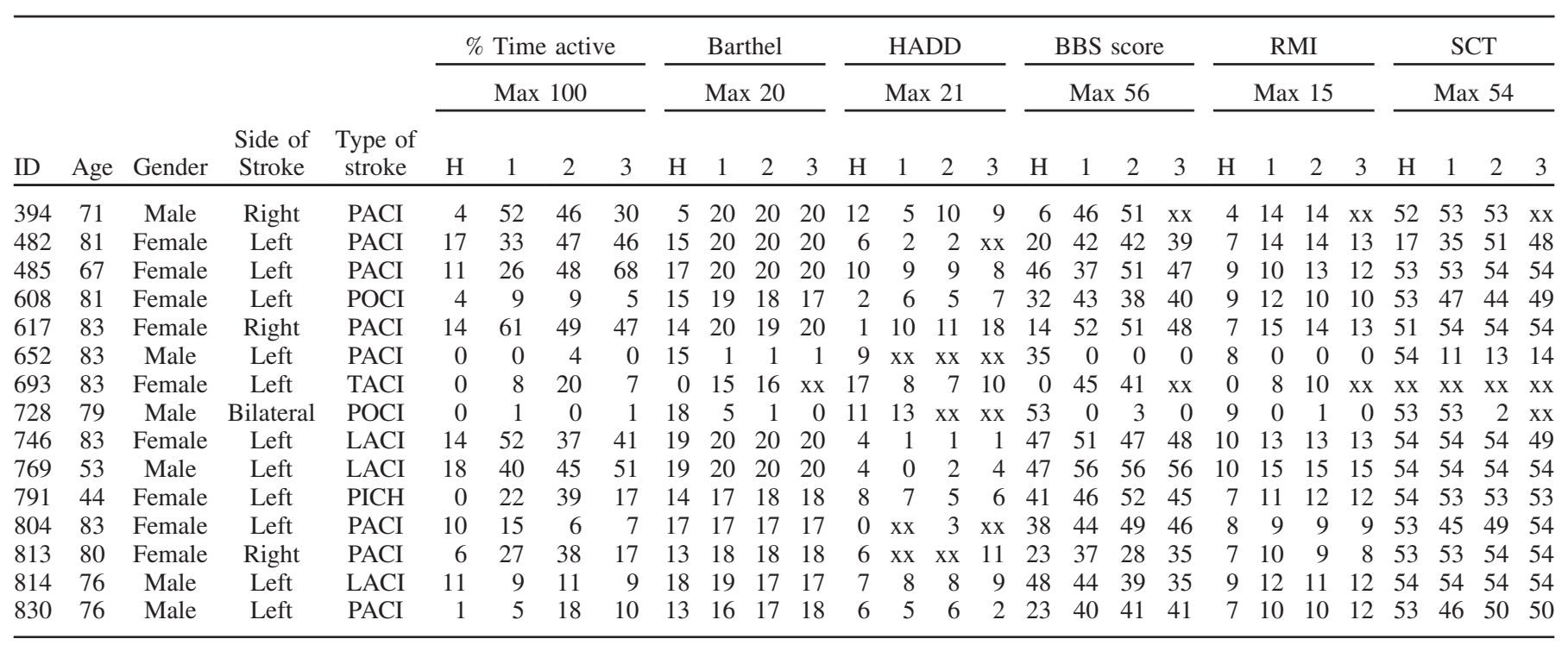

$\mathrm{xx}$ indicates missing data.

Higher Scores = better ability for Barthel, Barthel Index score; BBS, Berg Balance; RMI, Rivermead Mobility Index; SCT, Star Cancellation Test: scores of 51 and below $=$ test failure/visual neglect; HADD, Hospital Anxiety and Depression Scale depression component. Scores of $8-10=$ borderline case of depression and scores of $11-21=$ depression.

In hospital, results suggest a correlation between activity levels and HAD depression scores $(r=-0.539, p=0.038)$. Data in Table 3 highlight that those who had higher HAD depression scores (indicative of low mood and depression) were significantly less active than those who did not show signs of depression. While activity scores remain lower for those with depression at Year 1, this difference does no longer reach significance, probably due to the high degree of variability within the sample.

Activity levels in hospital did not correlate to balance, mobility, neglect or side of infarction. At Year $1(r=-0.632$, $p=0.011)$ and Year $2(r=-0.584, p=0.022)$, side of infarction was correlated to activity levels. Those with a right sided infarction were more active than those with a left-sided infarction. While this difference is still apparent on visual examination at Year 3 (Table 3), this does no longer reach significance. Similarly, those who had visual neglect were less active but this reached significance at Year $2(r=0.665, p=0.009)$ only.

Finally, correlational analysis suggests that those with better balance scores $(r=0.609, \quad p=0.015)$ and mobility scores $(r=0.729, p=0.002)$ were more active at Year 1 (data presented), Y2 and Y3 (similar r-levels and $p$ values but not shown, see Table 3 for activity level data).

\section{Discussion}

This is the first study to repeatedly explore change in activity levels among consecutively admitted randomly allocated stroke patients (from one distinct geographical area) over a period of 3 years post-stroke onset. We succeeded in recruiting a larger sample with the intention to undertake predictive analysis to identify the factors that have the greatest impact on activity levels. However, as a result of serious misconduct a large amount of data had to be excluded from analysis. This resulted in a very small complete data set and prevented in-depth statistical analysis.

We found that activity levels were low at all-time points poststroke. Direct comparison to other studies is difficult as most did not use the same device but using the reported activity counts, it appears that our sample was less active in comparison to agematched control data presented in other studies [13,14,33,34]. As no study to date has followed the same cohort of stroke 
Table 3. Time active in relation to side of stroke, measures of independence, balance, mood, mobility and neglect for complete cases.

\begin{tabular}{|c|c|c|c|c|c|c|c|c|c|c|c|c|c|c|}
\hline $\begin{array}{l}\text { Time active } \\
\text { (upright) } \%\end{array}$ & $\mathrm{~L}$ & $\mathrm{R}$ & $\leq 10$ & $\geq 11$ & $\leq 3$ & $\geq 4$ & $\leq 44$ & $\geq 45$ & $\leq 7$ & $\geq 8$ & $\leq 9$ & $\geq 10$ & $\leq 50$ & $\geq 51$ \\
\hline Year 2 & $\begin{array}{l}20.4 \\
4-48\end{array}$ & $\begin{array}{c}45.9 \\
38-50\end{array}$ & $\begin{array}{c}2.2 \\
0.2-4.3\end{array}$ & $\begin{array}{l}38.1 \\
6-50\end{array}$ & $\begin{array}{l}2.2 \\
0-4\end{array}$ & $\begin{array}{c}38 \\
6-50\end{array}$ & $\begin{array}{c}14.5 \\
0.2-47\end{array}$ & $\begin{array}{l}38.6 \\
6-50\end{array}$ & $\begin{array}{l}28.6 \\
6-47\end{array}$ & $\begin{array}{c}46.9 \\
11-49\end{array}$ & $\begin{array}{c}5.3 \\
0.2-38\end{array}$ & $\begin{array}{l}38.8 \\
9-50\end{array}$ & $\begin{array}{c}6.3 \\
0.2-18\end{array}$ & $\begin{array}{c}44.6 \\
11-50\end{array}$ \\
\hline Year 3 & $\begin{array}{c}9.8 \\
0-68\end{array}$ & $\begin{array}{c}30.2 \\
17-47\end{array}$ & $\begin{array}{l}0.3 \\
0-1\end{array}$ & $\begin{array}{l}16.9 \\
6-68\end{array}$ & $\begin{array}{l}0.3 \\
0-1\end{array}$ & $\begin{array}{c}17 \\
6-68\end{array}$ & $\begin{array}{c}8.9 \\
0-46\end{array}$ & $\begin{array}{l}43.9 \\
7-68\end{array}$ & $\begin{array}{l}16.9 \\
5-51\end{array}$ & $\begin{array}{c}23.9 \\
7-68\end{array}$ & $\begin{array}{c}7.5 \\
0-17\end{array}$ & $\begin{array}{l}43.5 \\
6-68\end{array}$ & $\begin{array}{c}9.8 \\
0-46\end{array}$ & $\begin{array}{l}16.9 \\
8-68\end{array}$ \\
\hline
\end{tabular}

${ }^{\text {a }}$ Score based on one participant only.

A, admission; D, discharge.

Barthel, Barthel Index score (higher score $=$ better ability, scores range from 0 to 20); FAC, Functional Ambulation Category (higher score $=$ better ability, scores range from 0 to 5); BBS, Berg Balance Scale (higher score = better ability, scores range from 0 to 56, scores of 45 and above are indicators of better balance ability). HADD, Hospital Anxiety and Depression Scale- depression component (higher score $=$ worse mood, scores range from 0 to 21, scores of 8 and above are considered and indicator of low mood). RMI, Rivermead Mobility Index (higher score $=$ better ability, scores range from 0 to 15, scores of 10 and above highlight independent mobility; SCT, Star Cancellation Test (higher scores $=$ better ability, scores of 50 and below are indicative of visual neglect).

Median scores and range are presented. Scores are presented for those who achieved higher or lower scores (split at the half way mark (for Barthel, FAC), according to the measures accepted cut-off points (HADD, SCT) and at points that have previously been highlighted at indicative of better or worse ability (BBS, RMI).

patients longitudinally over this length of time and even the stroke specific studies used a variety of different measurement tools, direct comparison to other studies is not possible. As step counts and time spent walking and the amount of time spent active are the descriptors that have been commonly described in other studies, we used these descriptors to compare findings in relation to the present study.

Hospitalised participants were engaged in activities for approximately $6 \%$ of the day. This percentage of time spent active was comparable to one study [6], higher than reported in another study [8] but lower than reported by Bernhardt et al. [7]. It would be possible to argue that differing findings can be explained as a result of differing recording techniques (observational versus body worn device) and that our sample was slightly older.

The most important observation from our study that agrees with the previous work is that (a) time spent upright and active in hospital is extremely low and (b) people with stroke are inactive for the majority of their waking day in hospital. On visual observation our findings suggest that activity levels improve in the first couple of years post-stroke and then slow or plateau at year 3, but the small number of complete cases and the high degree of variability within this small sample prevents us from making more definitive conclusions. Activity levels in our study are also considerably lower in comparison to similar age group of healthy adults [28] and a group of people with stroke who range in stroke onset from 3 to 20 months [31]. Both of these studies also used the activPAL, however are findings might differ because these researchers collected activity data (mean number of steps per day) over a 7-d period.

Most other community samples included people from 6 months to many years' post-stroke, used different devices and sometimes the time post-stroke was not presented [9-14]. Therefore, direct comparison to the findings in our study is not possible. The average number of steps reported between years 1 and 3 in the current study averaged around 4-5 steps/min (which translates into 1920-2400 steps per 8-h recording), which is similar to the step range (1389-2837) reported by others [9-11]. Two studies reported on time spent active among people who had their stroke an average 3 years ago $[12,13]$. In one of those studies participants spent less time active $(13 \%)$ while the sample in the other study was more active than our participants (35\% time spent active of which $19 \%$ in standing and $16 \%$ walking).

Although it could be argued that physical activity declines naturally with increasing age [35], a meta-analysis of 42 studies representing 6199 healthy subjects and a study exploring activity levels in older people using the activPAL both suggested that subjects aged 65 years or older still walk on over 6000 steps/d $[28,34]$ which is nearly 3 times higher than the highest number of average steps achieved at any time point in the current study. The level of low activity among healthy older people is of great concern to health care agencies [35] and therefore the significant level of inactivity among people with stroke in this study is of particularly concern.

Correlational analysis revealed several factors that might impact on activity levels including level of independence (Barthel), walking independence (FAC), depression (HAD), side of infarction, mobility and balance. Visual observations and correlational analysis are not very robust and caution is advised when attempting to generalise these findings. We have not attempted to identify any causal relationships in this study; we merely report for example those who are more independent spent more time engaged in upright activities or vice versa. Furthermore, the fact that correlations were inconsistent and only apparent at some time points (apart from Barthel and FAC) can be due to the variability within the sample and the small sample size, but it might be also an indicator of a chance findings. Before more definitive conclusions can be drawn, predictive analysis in a larger sample has to be undertaken to accept or reject the findings reported in this study. Taking into account these limitations, we do suggest that future studies take note of side of infarction, include measures of mobility, balance, mood and attention as these factors might be related to activity levels. It would be relevant in clinical practice, if we can identify the factors that impact most on activity levels either on admission to support decision making with regards to prognosis and discharge or at time of discharge to predict long-term activity levels to identify those most at need of additional interventions in the 
community. Other researchers have also reported that functional health and cognitive status were the most important predictors for physical activity participation [36].

There are other limitations of this study. First, it would have been preferable to apply activity monitors for a minimum of $24 \mathrm{~h}$ and ideally for 3 or $7 \mathrm{~d}$ [16] Due to pragmatic reasons (available staff and activity monitor resources) this was not possible and limits the generalizability of the findings. Furthermore, the monitoring sessions varied between assessments and activity counts had to be converted into the percentage of time spent doing an activity to allow us compare our findings to other studies who assessed activity levels over longer time periods, further limiting comparability and generalizability of our results. Second, the large amount of missing data and resulting small sample with complete data in this study was the main factor limiting the amount of analysis and conclusions that can be drawn from our findings. Despite these limitations, our findings though confirm that people with stroke living in the community are on the whole very inactive and would benefit from any intervention that promotes physical activity, for example those recommended by the National Institute for Health and Clinical Excellence [37]. Future studies are warranted to undertake a more in-depth statistical analysis to explore factors that predict change in activity status long term among a larger sample of people with complete data sets, measured over a longer period of time. Researchers also need to identify which interventions are most effective, cost effective and acceptable to people with chronic stroke. Best practice guidelines on how to set up stroke specific exercise programmes and services in the community have been developed [38]. We conclude that time spent standing and walking among people with stroke is very low. Time spent upright improved significantly by 1 year post-stroke; improvements appeared to slow down thereafter. Poor activity levels correlated with physical and psychological measures but larger studies are indicated to identify the most important predictors of activity levels.

\section{Declaration of interest}

The study was supported by a grant from The Stroke Association. The authors report no declarations of interest.

\section{References}

1. Robison J, Wiles R, Ellis-Hill C, et al. Resuming previously valued activities post stroke: who or what helps? Dis Rehabil 2009;31: 1555-66.

2. Ivey FM, Macko RF, Ryan AS, Hafer-Macko CE. Cardiovascular health and fitness after stroke. Top Stroke Rehabil 2005;12:1-16.

3. Evenson KR, Rosamond WD, Cai J, et al. Physical activity and ischemic stroke risk: the atherosclerosis risk in community study. Stroke 1999;30:1333-9.

4. Lee CDE, Folsom ARMD, Blair SNPED. Physical activity and stroke risk: a meta-analysis. Stroke 2003;34:2475-81.

5. Wendel-Vos GCW, Schuit AJ, Feskens EJM, et al. Physical activity and stroke. A meta-analysis of observational data. Int J Epidemiol 2004;33:787-98.

6. Egerton T, Maxwell DJ, Granat MH. Mobility activity of stroke patients during inpatient rehabilitation. Hong Kong Physiother J 2006;24:8-15.

7. Bernhardt J, Dewey H, Thrift A, Donnan G. Inactive and alone: physical activity within the first 14 days of acute stroke unit care. Stroke 2004;35:1005-9.

8. Lincoln NB, Willis D, Philips SA, et al. Comparison of rehabilitation practice on hospital wards for stroke patients. Stroke 1996;27: $18-23$.

9. Michael K, Macko RF. Ambulatory activity intensity profiles, fitness and fatigue in chronic stroke. Top Stroke Rehabil 2007;14: $5-12$.
10. Michael KM, Allen JK, Macko RF. Reduced ambulatory activity after stroke: the role of balance, gait, and cardiovascular fitness. Arch Phys Med Rehabil 2005;86:1552-6.

11. Michael K, Goldberg AP, Treuth MS, et al. Progressive adaptive physical activity in stroke improves balance, gait and fitness: preliminary results. Top Stroke Rehabil 2009;16:133-9.

12. Rand D, Eng JJ, Tang P-F, et al. How active are people with stroke? Use of accelerometers to assess physical activity. Stroke 2009;40: $163-8$.

13. Alzahrani MA, Ada L, Dean CM. Duration of physical activity is normal but frequency is reduced after stroke: an observational study. J Physiother 2011;57:47-51.

14. Manns PJ, Tomczak CR, Jelani A, et al. Use of the continous scale physical functional performance test in stroke survivors. Arch Phys Med Rehabil 2009;90:488-93.

15. Berlin J, Storti KL, Brach JS. Using activity monitors to measure physical activity in free-living conditions. Phys Ther 2006;86: 1137-45.

16. Ward DS, Evenson KR, Vaughn A, et al. Accelerometer use in physical activity: best practices and research recommendations. Med Sci Sports Exerc 2005;37:S582-8.

17. Valanou EM, Bamia C, Trichopoulou A. Methodology of physicalactivity and energy-expenditure assessment: a review. J Pub Health 2006;2:58-65.

18. Copeland JL, Eslinger DW. Acceleremoter assessment of physical activity in active, healthy older adults. J Aging Phys Act 2009;17: $17-30$.

19. Le Masurier GC, Tudor-Locke C. Comparison of pedometer and accelerometer accuracy under controlled conditions. Med Sci Sports Exerc 2003;35:867-71.

20. Maddocks M. Validity of three accelerometers during treadmill walking and motor vehicle travel. Br J Sports Med 2010;44: 606-8.

21. Huddleston J, Alaiti A, Goldvasser D, et al. Ambulatory measurement of knee motion and physical activity: preliminary evaluationof a smart activity monitor. J Neuroeng Rehabil 2006;3: $21-33$.

22. Tryon WW, Williams R. Fully proportional actigraphy: a new instrument. Behav Res Meth Instrum Comput 1996;28:392-403.

23. Heil DP. Predicting activity energy expenditure using the Actical activity monitor. Res Q Exerc Sport 2006;77:64-80.

24. Grant M, Dall P, Mitchell S, Granat M. Activity-monitor accuracy in measuring step number and cadence in community-dwelling older adults. J Aging Phys Activity 2008;16:201-14.

25. Godfrey A, Culhane KM, Lyons GM. Comparison of the performance of the activPAL Professional physical activity logger to a discrete accelerometer-based activity monitor. Med Eng Phys 2007; 29:930-4.

26. Grant PM, Cormac GR, Tigbe WW, Granat MH. The validation of a novel activity monitor in the measurement of posture and motion during everyday activities. Br J Sports Med 2006;40: 992-7.

27. Ryan CG, Grant PM, Tigbe WW, Granat MH. The validity and reliability of a novel activity monitor as a measure of walking. Brit J Sports Med 2006:40:779-84.

28. Lord S, Chastin SFM, McInnes L, et al. Exploring patterns of daily physical and sedentary behaviour in community-dwelling older adults. Age Ageing 2011;40:205-10.

29. Gebruers N, Vanroy C, Truijen S, et al. Monitoring of physical activity after stroke: a systematic review of accelerometry-based measures. Arch Phys Med Rehabil 2010;91:288-97.

30. Taraldsen K, Askim T, Sletvold O, et al. Evaluation of a body-worn sensor system to measure physical activity in older people with impaired function. Phys Ther 2011;91:277-85.

31. Touillet A, Guesdon H, Bosser G, et al. Assessment of compliance with prescribed activity by hemiplegic stroke patients after an exercise programme and physical activity education. Ann Phys Rehabil Med 2010;53:250-65.

32. Pickering RM, Kunkel D, Fitton C, et al. Stroke Association Rehabilitation and Research Centre Team. In-hospital recruitment to observational studies of stroke. Int J Rehabil Res 2010;33: $56-63$.

33. Busse ME, Pearson OR, Van Deursen R, Wiles CM. Quantified measurement of activity provides insight into motor function and recovery in neurological disease. J Neurol Neurpsych Psych 2004; 75:884-8. 
34. Bohannon RW. Number of pedometer-assessed steps taken per day by adults: a descriptive meta-analysis. Phys Ther 2007;87: 1642-50.

35. National Centre for Social Research. (2004). Health Survey for England; 2003. Available from: http://www.dh.gov.uk/en/ Publicationsandstatistics/Publications/PublicationsStatistics/DH 4098712 [last accessed 2 Jan 2012].

36. Külzer AM, Collor Scolari C, Gus M. Relationship between usual relationship between usual physical, cognitive and social activities and functional recovery at hospital discharge after acute stroke. J Rehabil Med 2008;40:195-9.
37. National Institute for Health and Clinical Excellence. Four commonly used methods to increase physical activity: brief interventions in primary care, exercise referral schemes, pedometers and community-based exercise programmes for walking and cycling; 2006. Available from: http://www.nice.org.uk/PH2 [last accessed 20 Dec 2011].

38. Best C, van Wijck F, Dinan-Young S, et al. Best practice guidance for the development of exercise after stroke services in community settings; November 2010. Available from: http://www.exerciseafterstroke.org.uk/ [last accessed 20 Dec 2011]. 